\title{
On the Origin of Dark Matter Axions
}

\author{
E.P.S. Shellard and R.A. Battye ${ }^{\text {a }}$ \\ a Department of Applied Mathematics and Theoretical Physics, University of Cambridge, \\ Silver Street, Cambridge, CB3 9EW, U.K.
}

\begin{abstract}
We discuss the possible sources of dark matter axions in the early universe. In the standard thermal scenario, an axion string network forms at the Peccei-Quinn phase transition $T \sim f_{\mathrm{a}}$ and then radiatively decays into a cosmological background of axions; to be the dark matter, these axions must have a mass $m_{\mathrm{a}} \sim 100 \mu \mathrm{eV}$ with specified large uncertainties. An inflationary phase with a reheat temperature below the PQ-scale $T_{\text {reh }} \lesssim f_{\mathrm{a}}$ can also produce axion strings through quantum fluctuations, provided that the Hubble parameter during inflation is large $H_{1} \gtrsim f_{\mathrm{a}}$; this case again implies a dark matter axion mass $m_{\mathrm{a}} \sim 100 \mu \mathrm{eV}$. For a smaller Hubble parameter during inflation $H_{1} \lesssim f_{\mathrm{a}}$, 'anthropic tuning' allows dark matter axions to have any mass in a huge range below $m_{\mathrm{a}} \lesssim 1 \mathrm{meV}$.
\end{abstract}

\section{INTRODUCTION}

The axion has remained a popular dark matter candidate because of its enduring motivation as an elegant solution to the strong CP-problem [1]. Despite early hopes of discovery, it turned out that in order to be consistent with accelerator searches and astrophysics, the axion must be nearly 'invisible' and extremely light. Its couplings and mass are inversely proportional to the (large) Peccei-Quinn scale $f_{\mathrm{a}}$ as in

$m_{\mathrm{a}}=6.2 \times 10^{-6} \mathrm{eV}\left(\frac{10^{12} \mathrm{GeV}}{f_{\mathrm{a}}}\right)$.

Accelerator constraints have been largely superseded by those from astrophysics; because the axion is so weakly coupled, volume effects can compete with other surface and convective stellar energy loss mechanisms. The strongest astrophysical constraints on the axion mass derive from studies of supernova $1987 \mathrm{a}$ and conservative estimates yield $m_{\mathrm{a}} \lesssim 10 \mathrm{meV}$ [2]. The present programme of large-scale axion search experiments [3] are sensitive to a mass range $m_{a} \sim 1-10 \mu \mathrm{eV}$, which has been chosen for a variety of historical and technological reasons. Our primary focus here, however, is not on constraints on the viable axion mass range, but rather on efforts to predict the mass of a dark matter axion from cosmology.

\section{STANDARD AXION COSMOLOGY}

The cosmology of the axion is determined by the two energy scales $f_{\mathrm{a}}$ and $\Lambda_{\mathrm{QCD}}$. The first important event is the Peccei-Quinn phase transition which is broken at a high temperature $T \sim f_{\mathrm{a}} \gtrsim 10^{9} \mathrm{GeV}$. This creates the axion, at this stage an effectively massless pseudo-Goldstone boson, as well as a network of axion strings 四 which decays gradually into a background cosmic axions [5]. (Note that one can engineer models in which an inflationary epoch interferes with the effects of the Peccei-Quinn phase transition, as we shall discuss in the next section.) At a much lower temperature $T \sim \Lambda_{\mathrm{QCD}}$ after axion and string formation, instanton effects 'switch on', the axions acquire a small mass, domain walls form [6] between the strings 沟 and the complex hybrid network annihilates into axions in about one Hubble time [7].

There are three possible mechanisms by which axions are produced in the 'standard thermal scenario': (i) thermal production, (ii) axion string radiation and (iii) hybrid defect annihilation when $T=\Lambda_{Q C D}$. Axions consistent with the astrophysical bounds must decouple from thermal equilibrium very early; their subsequent history and number density is analogous to the decoupled neutrino, except that unlike a $100 \mathrm{eV}$ massive neutrino, thermal axions cannot hope to dominate 
the universe with $m_{\mathrm{a}} \lesssim 10 \mathrm{meV}$. We now turn to the two dominant axion production mechanisms, but first we address an important historical digression.

\subsection{Misalignment misconceptions}

The original papers on axions suggested that axion production primarily occurred, not through the above mechanisms, but instead by 'misalignment' effects at the QCD phase transition [8]. Before the axion mass 'switches on', the axion field $\theta$ takes random values throughout space in the range 0 to $2 \pi$; it is the phase of the PQfield lying at the bottom of a $U(1)$ 'Mexican hat' potential. However, afterwards the potential becomes tilted and the true minimum becomes $\theta=0$, so the field in the 'misalignment' picture begins to coherently oscillate about this minimum; this homogeneous mode corresponds to the 'creation' of zero momentum axions. Given an initial rms value $\theta_{\mathrm{i}}$ for these oscillations, it is relatively straightforward to estimate the total energy density in zero momentum axions and compare these to the present mass density of the universe (assuming a flat $\Omega=1 \mathrm{FRW}$ cosmology) [8,9]:

$\Omega_{\mathrm{a}, \text { hom }} \approx 2 \Delta h^{-2} \theta_{\mathrm{i}}^{2} \mathrm{f}\left(\theta_{\mathrm{i}}\right)\left(\frac{10^{-6} \mathrm{eV}}{m_{\mathrm{a}}}\right)^{1.18}$

where $\Delta \approx 3^{ \pm 1}$ accounts for both modeldependent axion uncertainties and those due to the nature of the QCD phase transition, and $h$ is the rescaled Hubble parameter. The function $f(\theta)$ is an anharmonic correction for fields near the top of the potential close to unstable equilibrium $\theta \approx \pi$, that is, with $\mathrm{f}(0)=1$ at the base $\theta \approx 0$ and diverging logarithmically for $\theta \rightarrow \pi$ 10]. If valid, the estimate (2) would imply a constraint $m_{\mathrm{a}} \gtrsim 5 \mu \mathrm{eV}$ for the anticipated thermal initial conditions with $\theta_{\mathrm{i}}=\mathcal{O}(1)[8,9$.

As applied to the thermal scenario, the expression (2) is actually a very considerable underestimate for at least two reasons: First, the axions are not 'created' by the mass 'switch on' at $t=t_{Q C D}$, they are already there with a specific momentum spectrum $g(k)$ determined by dynamical mechanisms prior to this time. The actual axion number obtained from $g(k)$ is much larger than the rms average assumed in (2) which ig- nores the true particle content. Secondly, this estimate was derived before much stronger topological effects were realized, notably the presence of axion strings and domain walls. In any case, these nonlinear effects complicate the oscillatory behaviour considerably, implying that the homogeneous estimate (2) is poorly motivated.

\subsection{Axion string network decay}

Axions and axion strings are inextricably intertwined. Like ordinary superconductors or superfluid ${ }^{4} \mathrm{He}$, axion models contain a broken $U(1)$ symmetry and so there exist vortex-line solutions. Combine this fact with the Peccei-Quinn phase transition, which means the field is uncorrelated beyond the horizon, and a random network of axion strings must inevitably form. An axion string corresponds to a non-trivial winding from 0 to $2 \pi$ of the axion field $\theta$ around the bottom of its 'Mexican hat' potential. It is a global string with long-range fields, so its energy per unit length $\mu$ has a logarithmic divergence which is cut-off by the string curvature radius $R \lesssim t$, that is, $\mu \approx 2 \pi f_{\mathrm{a}}^{2} \ln (t / \delta)$, where the string core width is $\delta \approx f_{\mathrm{a}}^{-1}$. The axion string, despite this logarithmic divergence, is a strongly localized object; if we have a string stretching across the horizon at the QCD temperature, then $\ln (t / \delta) \sim 65$ and over $95 \%$ of its energy lies within a tight cylinder enclosing only $0.1 \%$ of the horizon volume. To first order, then, the string behaves like a local cosmic string, a fact that can be established by a precise analytic derivation and careful comparison with numerical simulations [11].

After formation and a short period of damped evolution, the axion string network will evolve towards a scale-invariant regime with a fixed number of strings crossing each horizon volume (for a cosmic string review see ref. [12]). This gradual demise of the network is achieved by the production of small loops which oscillate relativistically and radiate primarily into axions. The overall density of strings splits neatly into two distinct parts, long strings with length $\ell>t$ and a population of small loops $\ell<t$, that is, $\rho=\rho_{\infty}+\rho_{L}$. High resolution numerical simulations confirm this picture of string evolution and suggest that the long string density during the ra- 
diation era is $\rho_{\infty} \approx 13 \mu / t^{2}$ 13]. To date, analytic descriptions of the loop distribution have used the well-known string 'one scale' model, which predicts a number density of loops defined as $\mu \ell n(\ell, t) d \ell=\rho_{L}(\ell, t) d \ell$ in the interval $\ell$ to $\ell+d \ell$ to be given by

$n(\ell, t)=\frac{4 \alpha^{1 / 2}(1+\kappa / \alpha)^{3 / 2}}{(\ell+\kappa t)^{5 / 2} t^{3 / 2}}$,

where $\alpha$ is the typical loop creation size relative to the horizon and $\kappa \approx 65 /[2 \pi \ln (t / \delta)]$ is the loop radiation rate 14. Once formed at $t=t_{0}$ with length $\ell_{0}$, a typical loop shrinks linearly as it decays into axions $\ell=\ell_{0}-\kappa\left(t-t_{0}\right)$. The key uncertainty in this treatment is the loop creation size $\alpha$, but compelling heuristic arguments place it near the radiative backreaction scale, $\alpha \sim \kappa$. (If this is the case, we note that the loop contribution is over an order of magnitude larger than direct axion radiation from long strings.)

String loops oscillate with a period $T=\ell / 2$ and radiate into harmonics of this frequency (labelled by $n$ ), just like other classical sources. Unless a loop has a particularly degenerate trajectory, it will have a radiation spectrum $P_{n} \propto n^{-q}$ with a spectral index $q>4 / 3$, that is, the spectrum is dominated by the lowest available modes. [7 Given the loop density (3), we can then calculate the spectral number density of axions $d n_{\mathrm{a}} / d \omega$, which turns out to be essentially independent of the exact loop radiation spectrum for $q>4 / 3$. From this expression we can integrate over $\omega$ to find the total axion number at the time $t_{\mathrm{QCD}}$, that is, when the axion mass 'switches on' and the string network annihilates. Subsequently, the axion number will be conserved, so we can find the number-to-entropy ratio and project forward to the present day. Multiplying the present number density by the axion mass $m_{\text {a }}$ yields the overall axion string contribution to the density of the universe 14]:

$\Omega_{\mathrm{a}, \text { string }} \approx 110 \Delta h^{-2}\left(\frac{10^{-6} \mathrm{eV}}{m_{\mathrm{a}}}\right)^{1.18} f(\alpha / \kappa)$,

\footnotetext{
${ }^{1}$ Historically, there has been some debate on the radiation spectrum issue but the reader is referred elsewhere for further details 15$]$.
}

where

$f(\alpha / \kappa)=\left[\left(1+\frac{\alpha}{\kappa}\right)^{3 / 2}-1\right]$.

The key additional uncertainty from the string model is the ratio $\alpha / \kappa \sim \mathcal{O}(1)$, which should be clearly distinguished from particle physics and cosmological uncertainties inherent in $\Delta$ and $h$ (which appear in all estimates of $\Omega_{\mathrm{a}}$ ). With a Hubble parameter near $h=0.5$, the string estimate (何) tends to favour a dark matter axion with a mass $m_{\mathrm{a}} \sim 100 \mu \mathrm{eV}$, as we shall discuss in the conclusion. A comparison with (2) confirms that $\Omega_{\mathrm{a}, \text { string }}$ is well over an order of magnitude larger than the 'misalignment' contribution.

\subsection{Hybrid defect annihilation}

Near the QCD phase transition the axion acquires a mass and network evolution alters dramatically because domain walls form. Large field variations around the strings collapse into these domain walls, which subsequently begin to dominate over the string dynamics. This occurs when the wall surface tension $\sigma$ becomes comparable to the string tension due to the typical curvature $\sigma \sim \mu / t$. The demise of the hybrid string-wall network proceeds rapidly, as demonstrated numerically [7]. The strings frequently intersect and intercommute with the walls, effectively 'slicing up' the network into small oscillating walls bounded by string loops. Multiple selfintersections will reduce these pieces in size until the strings dominate the dynamics again and decay continues through axion emission.

An order-of-magnitude estimate of the demise of the string-domain wall network indicates that there is an additional contribution 16]

$\Omega_{\mathrm{a}, \mathrm{dw}} \sim \mathcal{O}(10) \Delta h^{-2}\left(\frac{10^{-6} \mathrm{eV}}{m_{\mathrm{a}}}\right)^{1.18}$.

This 'domain wall' contribution is ultimately due to loops which are created at the time $\sim t_{\mathrm{QCD}}$. Although the resulting loop density will be similar to (3), there is not the same accumulation from early times, so it is likely to be subdominant 14 relative to (4). More recent work, 17 questions this picture by suggesting that the walls stretching between long strings dominate and will pro- 
duce a contribution anywhere in the wide range $\Omega_{\mathrm{a}, \mathrm{dw}} \sim(1-44) \Omega_{\mathrm{a}, \mathrm{string}}$; however, this assertion requires stronger quantitative support. Overall, like most effects.2 the domain wall contribution will serve to further strengthen the string bound (4) on the axion.

Up to this point we have only considered the simplest axion models with a unique vacuum $N=1$, so what happens when $N>1$ ? In this case, any strings present become attached to $N$ domain walls at the QCD-scale. Such a network 'scales' rather than annihilates, and so it is cosmologically disastrous being incompatible (at the very least) with $\mathrm{CMB}$ isotropy.

\section{INFLATIONARY AXION MODELS}

The relationship between inflation and dark matter axions is rather mysterious. Its significance depends on the magnitude of the Peccei-Quinn scale $f_{\text {a }}$ relative to two key inflationary parameters, (i) the reheat temperature of the universe $T_{\text {reh }}$ at the end of inflation and (ii) the Hubble parameter $H_{1}$ as the observed universe first exits the horizon during inflation. Inflation is irrelevant to the axion if $T_{\text {reh }} \gtrsim f_{\text {a }}$ because, in this case, the PQ-symmetry is restored and the universe returns to the 'standard thermal scenario' in which axion strings form and the estimate (伍) pertains. Consider, then, the two inflationary axion scenarios with $T_{\text {reh }} \lesssim f_{\text {a }}$.

\subsection{Anthropic misalignment and quantum fluctuations (Case 1: $H_{1}<f_{\mathrm{a}}$ )}

In an inflationary model for which $f_{\mathrm{a}}>H_{1}>$ $T_{\text {reh }}$, the $\theta$-parameter or axion angle will be set homogeneously over large inflationary domains before inflation finishes 18. In this case, the whole observable universe emerges from a single Hubble volume in which this parameter has some fixed initial value $\theta_{\mathrm{i}}$. Because the axion remains out of thermal equilibrium for large $f_{\mathrm{a}}$, subse-

\footnotetext{
${ }^{2}$ We note briefly that it is also possible to weaken any axion mass bound through catastrophic entropy production between the QCD-scale and nucleosynthesis, that is, in the timescale range $10^{-4} s \lesssim t_{\text {ent }} \lesssim 10^{-2} s$. Usually this involves the energy density of the universe becoming temporarily dominated by an exotic massive particle with a tuned decay timescale.
}

quent evolution and reheating does not disturb $\theta_{\mathrm{i}}$ until the axion mass 'switches on' at $T \sim \Lambda_{\mathrm{QCD}}$. Afterwards, the field begins to oscillate coherently, because it is misaligned by the angle $\theta_{\mathrm{i}}$ from the true minimum $\theta=0$. This homogeneous mode corresponds to a background of zero momentum axions and it is the one circumstance under which the misalignment formula (2) actually gives an accurate estimate of the relative axion density $\Omega_{\mathrm{a}}$.

By considering the dependence $\Omega_{\mathrm{a}, \mathrm{h}} \propto \theta_{\mathrm{i}}^{2}$ in (2), we see that inflation models have an intrinsic arbitrariness given by the different random magnitudes of $\theta_{\mathrm{i}}$ in different inflationary domains [18]. While a large value of $f_{\mathrm{a}} \gg 10^{12} \mathrm{GeV}$ might have been thought to be observationally excluded, it can actually be accommodated in domains where $\theta_{\mathrm{i}}<<1$. This may seem highly unlikely but, if we consider an infinite inflationary manifold or a multiple universe scenario including 'all possible worlds', then life as we know it would be excluded from those domains with large $\theta_{\mathrm{i}}=\mathcal{O}(1)$ because the baryon-to-axion ratio would be too low 19. Thus, accepting this anthropic selection effect, we have to concede that axions could be the dark matter $\Omega_{\mathrm{a}} \approx 1$ if we live in a domain with a 'tuned' $\theta$-parameter?:

$\theta_{\mathrm{i}} \approx 0.3 \Delta^{-1 / 2} h\left(\frac{m_{\mathrm{a}}}{10^{-6} \mathrm{eV}}\right)^{0.6}$

For $\theta_{\mathrm{i}} \approx \mathcal{O}(1)$, this suggests an axion with $m_{\mathrm{a}} \sim$ $5 \mu \mathrm{eV}(h=0.5)$, though actually inflation makes no definite prediction from (7) beyond apparently specifying $m_{\mathrm{a}} \gtrsim 10 \mu \mathrm{eV}$. But even this restriction is not valid; if we observe (2) carefully we see that we can also obtain a dark matter axion for higher $m_{\mathrm{a}}$ by fine-tuning $\theta_{\mathrm{i}}$ near $\pi$. The anharmonic term $\mathrm{f}(\theta)$ with an apparent logarithmic divergence allows $\Omega_{\mathrm{a}} \approx 1$ for a much heavier dark matter axion 10 .

This simple 'anthropic tuning' picture is significantly altered by quantum effects. Like any minimally coupled massless field during inflation, the axion will have a spectrum of quantum excitations associated with the Gibbons-Hawking temperature $T \sim H / 2 \pi$. This implies the field

\footnotetext{
${ }^{3}$ This is not quite in the spirit of the original motivation for the axion!
} 
will acquire fluctuations about its mean value $\theta_{\mathrm{i}}$ of magnitude $\delta \theta=H / 2 \pi f_{\mathrm{a}}$, giving an effective rms value $\theta_{\text {eff }}^{2}=\left(\theta_{\mathrm{i}}+\delta \theta\right)^{2}$. Even if our universe began in an inflationary domain with $\theta_{\mathrm{i}}=0$, there will be a minimum misalignment angle set by $\delta \theta$; this implies that we cannot always finetune $\theta_{\mathrm{i}}$ in (7) such that $\Omega_{a} \lesssim 1$. Worse still, the fluctuations $\delta \theta$ imply isocurvature fluctuations in the axion density [20] given by (2), that is, $\delta \rho_{\mathrm{a}} \propto \theta_{\text {eff }}^{2} \approx \theta_{\mathrm{i}}^{2}+2 \theta_{\mathrm{i}} \delta \theta+\delta \theta^{2}$. Such density fluctuations will also create cosmic microwave background (CMB) anisotropies which are strongly constrained, $\delta T / T \sim \delta \rho / \rho \sim 2 \delta \theta / \theta_{\text {eff }} \lesssim 10^{-5}$. Combining the quantum fluctuation $\delta \theta$ with the $\theta$-requirement for a dark matter axion (7), we obtain a strong constraint on the Hubble parameter during inflation 21

$$
H_{1} \lesssim 10^{9} \mathrm{GeV}\left(\frac{10^{-6} \mathrm{eV}}{m_{\mathrm{a}}}\right)^{0.4},
$$

which is valid for small masses $m_{\mathrm{a}} \lesssim 10 \mu \mathrm{eV}$. Here, $H_{1}$ is the Hubble parameter as fluctuations associated with the time when the microwave anisotropies first leave the horizon some 50-60 efoldings before the end of inflation. We conclude from (8) that inflation in the $f_{\mathrm{a}}>H_{1}>T_{\text {reh }}$ regime must have a small Hubble parameter $H_{1}$ or the dark matter axion must be extremely light, $m_{\mathrm{a}} \ll 1 \mu \mathrm{eV}$. Inflation now has many guises and models exist with Hubble parameters anywhere in the range $H_{1} \sim 10^{2}-10^{14} \mathrm{GeV}$, so a detectable inflationary axion with $m_{\mathrm{a}} \sim 1 \mu \mathrm{eV}$ is possible in principle. Note, however, that the constraint (8) can be circumvented in more complicated multifield inflation models 22,

Quantum effects also constrain the 'anthropic tuning' $\theta_{\mathrm{i}} \rightarrow \pi$ which is required for a larger mass axion $m_{\mathrm{a}} \gtrsim 10 \mu \mathrm{eV}$. For simple inflation models, excessive isocurvature fluctuations imply the dark matter axion mass is bounded above by $m_{\mathrm{a}} \lesssim 1 \mathrm{meV}$ for $H_{1} \gtrsim 10^{2} \mathrm{GeV}[10$. Note that this is a considerably heavier dark matter axion than the oft-quoted inflationary limit $m_{\mathrm{a}} \lesssim 5 \mu \mathrm{eV}$.

\subsection{Axion string network creation during inflation (Case 2: $H_{1} \gtrsim f_{\mathrm{a}}$ )}

Even for a low reheat temperature $T_{\text {reh }}<f_{\mathrm{a}}$, one can envisage inflation models with a large
Hubble parameter during inflation $H_{1} \gtrsim f_{\mathrm{a}}$ (such as chaotic inflation with $H_{1} \sim 10^{13}-10^{14} \mathrm{GeV}$ ). The quantum fluctuations in this case are sufficient to take the Peccei-Quinn field over the top of the potential leaving large spatial variations and topologically non-trivial windings [23]. We can interpret this as the Gibbons-Hawking temperature 'restoring' the $\mathrm{PQ}$-symmetry $T_{\mathrm{GH}} \gtrsim f_{\mathrm{a}}$. As inflation draws to a close and $H$ falls below $f_{\mathrm{a}}$, these fluctuations will become negligible and a string network will form. Provided inflation does not continue beyond this point for more than about another 30 e-foldings, we will effectively return to the 'standard thermal' scenario in which axions are produced by a decaying string network. So such low reheat inflation models are again only compatible with a dark matter axion, $m_{\mathrm{a}} \approx 100 \mu \mathrm{eV}$.

We note that there is also a borderline scenario with $H_{1} \sim f_{\mathrm{a}}$ and in which domain walls form but few strings. Since strings are required to remove them, such domian walls will be cosmologically unacceptable 24.

\section{CONCLUSIONS}

We have endeavoured to provide an overview of axion cosmology focussing on the mass of a dark matter axion. First, the cosmological axion density was considered in the standard thermal scenario where the dominant contribution comes from axion strings. In this case there is, in principle, a well-defined calculational method to precisely predict the mass $m_{\mathrm{a}}$ of a dark matter axion. For the currently favoured value of the Hubble parameter $\left(H_{0} \approx 60 \mathrm{~km} \mathrm{~s}^{-1} \mathrm{Mpc}^{-1}\right)$, the estimate (1) predicts a dark matter axion of mass $m_{\mathrm{a}} \approx 200 \mu \mathrm{eV}$, where significant uncertainties from all sources approach an order of magnitude. The additional uncertainty in this string calculation is the parameter ratio $\alpha / \kappa \approx 1$, that is, the ratio of the loop size to radiation backreaction scale.

Secondly, we have reviewed inflationary axion cosmology showing that (i) many inflation models return us to the standard thermal scenario with $m_{\mathrm{a}} \sim 100 \mu \mathrm{eV}$, (ii) some inflation models are essentially incompatible with a detectable dark 
matter axion and (iii), because of the possibility of 'anthropic fine-tuning', other inflation models can be constructed which incorporate a dark matter axion mass anywhere below $m_{\mathrm{a}} \lesssim 1 \mathrm{meV}$.

We conclude that, while a dark matter axion might possibly lurk anywhere in an enormous mass range below $m_{\mathrm{a}} \lesssim 1 \mathrm{meV}$, the bestmotivated mass for future axion searches lies near $m_{\mathrm{a}} \sim 100 \mu \mathrm{eV}$, a standard thermal scenario prediction which is also compatible with a broad class of inflationary models.

\section{ACKNOWLEDGEMENTS}

We are grateful to Pierre Sikivie for his kind invitation to attend the Florida Axions Workshop, at which we benefitted from many informative discussions with members of the theoretical and experimental axion community. We acknowledge useful conversations with Andrei Linde, David Lyth and Georg Raffelt. This work is supported by PPARC.

\section{REFERENCES}

1. R.D. Peccei and H.R. Quinn, Phys. Rev. Lett. 38, 1440 (1977); Phys. Rev. D16, 1791. F. Wilczek,, Phys. Rev. Lett. 40, 279 (1978). S. Weinberg, Phys. Rev. Lett. 40, 223 (1978).

2. G.G. Raffelt (1997), astro-ph/9707268.

3. C. Hagmann et al (1998), astro-ph/9802061. I. Ogawa, S. Matsuki \& K. Yamamoto, Phys. Rev. D53, R1740 (1996). Refer to other papers in these proceedings.

4. A. Vilenkin and A.E. Everrett, Phys. Rev. Lett. 48, 1867 (1982).

5. R.L. Davis, Phys. Lett. 180B, 225 (1986). R.L. Davis and E.P.S. Shellard, Nucl. Phys. B324, 167 (1989).

6. P. Sikivie, Phys. Rev. Lett. 48, 1156 (1982).

7. E.P.S. Shellard (1986), in Proceedings of the 26th Liege International Astrophysical Colloquium,, Demaret, J., ed. (University de Liege). E.P.S. Shellard (1990), 'Axion strings and domain walls', in Formation and Evolution of Cosmic Strings, Gibbons, G.W., Hawking, S.W., \& Vachaspati, V., eds. (Cambridge University Press).
8. J. Preskill, M.B. Wise and F. Wilczek, Phys. Lett. 120B, 127 (1983). L. Abbott \& P. Sikivie, Phys. Lett. 120B, 133 (1983). M. Dine \& W. Fischler, Phys. Lett. 120B, 137 (1983).

9. M.S. Turner, Phys. Rev. D33, 889 (1986).

10. E.P.S Shellard and R.A. Battye (1998), Cosmic Axions astro-ph/9802216 E.P.S. Shellard \& R.A. Battye (1998), 'Inflationary axion cosmology revisited', DAMTP preprint.

11. R.L. Davis and E.P.S. Shellard, Phys. Lett. 214B, 219 (1988). R.A. Battye and E.P.S. Shellard, Nucl. Phys. B423, 260 (1994).

12. A. Vilenkin \& E.P.S. Shellard (1994), Cosmic strings and other Topological Defects (Cambridge University Press).

13. D.P. Bennett and F.R. Bouchet, Phys. Rev. D41, 2408 (1990). B. Allen and E.P.S. Shellard, Phys. Rev. Lett. 64, 119 (1990).

14. R.A. Battye and E.P.S Shellard, Phys. Rev. Lett. 73, 2954 (1994). Erratum: Phys. Rev. Lett. 76, 2203.

15. R.A. Battye and E.P.S Shellard (1998), 'Spectrum of radiation from axion strings' in these proceedings.

16. D.H. Lyth, Phys. Lett. 275B, 279 (1992).

17. M. Nagasawa, Prog. Theor. Phys. 98, 851 (1997). M. Nagasawa \& M. Kawasaki, Phys. Rev. D50, 4821 (1994).

18. S.Y-. Pi, Phys. Rev. Lett. 52, 1725 (1984).

19. A.D. Linde, Phys. Lett. 201B, 437 (1988).

20. M.S. Turner, F. Wilczek \& A. Zee, Phys. Lett. 120B, 127 (1983).

21. D.H. Lyth, Phys. Lett. B236, 408 (1990). M.S. Turner \& F. Wilczek, Phys. Rev. Lett. 66, 5 (1991).

22. A.D. Linde, Phys. Lett. 259B, 38 (1991).

23. D.H. Lyth and E.D. Stewart, Phys. Lett. 283B, 189 (1992).

24. A.D. Linde \& D.H. Lyth, Phys. Lett. 246B, 353 (1990). 